\title{
Regularity of solutions to anisotropic nonlocal equations
}

\author{
Jamil Chaker ${ }^{1}$
}

Received: 27 April 2018 / Accepted: 18 December 2019 / Published online: 28 January 2020

(c) The Author(s) 2020

\begin{abstract}
We study harmonic functions associated to systems of stochastic differential equations of the form $d X_{t}^{i}=A_{i 1}\left(X_{t-}\right) d Z_{t}^{1}+\cdots+A_{i d}\left(X_{t-}\right) d Z_{t}^{d}, i \in\{1, \ldots, d\}$, where $Z_{t}^{j}$ are independent one-dimensional symmetric stable processes of order $\alpha_{j} \in(0,2), j \in\{1, \ldots, d\}$. In this article we prove Hölder regularity of bounded harmonic functions with respect to solutions to such systems.
\end{abstract}

Keywords Jump processes · Harmonic functions · Hölder continuity · Support theorem · Anisotropy $\cdot$ Nonlocal Operators

Mathematics Subject Classification Primary 60J75; Secondary 60H10 · 31B05 · 60G52

\section{Introduction}

The consideration of stochastic processes with jumps and anisotropic behavior is natural and reasonable since such objects arise in several natural and financial models. In certain circumstances Lévy processes with jumps are more suitable to capture empirical facts that diffusion models do. See for instance [14] for examples of financial models with jumps.

In the nineteen fifties, De Giorgi [15] and Nash [29] independently prove an a-priori Hölder estimate for weak solutions $u$ to second order equations of the form

$$
\operatorname{div}(A(x) \nabla u(x))=0
$$

for uniformly elliptic and measurable coefficients A. In [28], Moser proves Hölder continuity of weak solutions and gives a proof of an elliptic Harnack inequality for weak solutions to this equation. This article provides a new technique of how to derive an a-priori Hölder estimate from the Harnack inequality. For a large class of local operators, the Hölder continuity can be derived from the Harnack inequality, see for instance [19]. For a comprehensive introduction into Harnack inequalities, we refer the reader e.g. to [20].

The corresponding case of operators in non-divergence form is treated in by Krylov and Safonov in [23]. The authors develop a technique for proving Hölder regularity and the

\footnotetext{
$凶 \quad$ Jamil Chaker

jchaker@math.uni-bielefeld.de

1 Universität Bielefeld, Fakultät für Mathematik, Postfach 100131, 33501 Bielefeld, Germany
} 
Harnack inequality for harmonic functions corresponding to non-divergence form elliptic operators. They take a probabilistic point of view and make use of the martingale problem to prove regularity estimates for harmonic functions. The main tool is a support theorem, which gives information about the topological support for solutions to the martingale problem associated to the corresponding operator. This technique is also used in [6] to prove similar results for nonlocal operators of the form

$$
L f(x)=\int_{\mathbb{R}^{d} \backslash\{0\}}\left[f(x+h)-f(x)-\mathbb{1}_{\{|h| \leq 1\}} h \cdot \nabla f(x)\right] a(x, h) d h
$$

under suitable assumptions on the function $a$. In [4] Bass and Chen follow the same ideas to prove Hölder regularity for harmonic functions associated to solutions of systems of stochastic differential equations driven by Lévy processes with highly singular Lévy measures. In this work we extend the results obtained by Bass and Chen to a larger class of driving Lévy processes.

A one-dimensional Lévy process $\left(Y_{t}\right)_{t \geq 0}$ is called symmetric stable processes of order $\epsilon$ $(0,2)$ if its characteristic function is given by

$$
\mathbb{E} e^{i \xi Y_{t}}=e^{-t|\xi|^{\gamma}}, \quad \xi \in \mathbb{R} .
$$

The Lévy measure of such a process is given by $v(d h)=c_{\gamma}|h|^{-1-\gamma} d h$, where $c_{\gamma}=$ $2^{\gamma} \Gamma\left(\frac{1+\gamma}{2}\right) /\left|\Gamma\left(-\frac{\gamma}{2}\right)\right|$.

Let $d \in \mathbb{N}$ and $d \geq 2$. We assume that $Z_{t}^{i}, i=1, \ldots, d$, are independent one-dimensional symmetric stable processes of order $\alpha_{i} \in(0,2)$ and define $Z=\left(Z_{t}\right)_{t \geq 0}=\left(Z_{t}^{1}, \ldots, Z_{t}^{d}\right)_{t \geq 0}$.

The Lévy-measure of this process is supported on the coordinate axes and is given by

$$
v(d w)=\sum_{k=1}^{d}\left(\frac{c_{\alpha_{k}}}{\left|w_{k}\right|^{1+\alpha_{k}}} d w_{k}\left(\prod_{j \neq k} \delta_{\{0\}}\left(d w_{j}\right)\right)\right) .
$$

Therefore $v(A)=0$ for every set $A \subset \mathbb{R}^{d}$, which has an empty intersection with the coordinate axes. The generator $L$ of $Z$ is given for $f \in C_{b}^{2}\left(\mathbb{R}^{d}\right)$ by the formula

$$
L f(x)=\sum_{k=1}^{d} \int_{\mathbb{R} \backslash\{0\}}\left(f\left(x+h e_{k}\right)-f(x)-\mathbb{1}_{\{|h| \leq 1\}} \partial_{k} f(x) h\right) \frac{c_{\alpha_{k}}}{|h|^{1+\alpha_{k}}} d h .
$$

For a deeper discussion on Lévy processes and their generators we refer the reader to [30].

Let $x_{0} \in \mathbb{R}^{d}$ and $A: \mathbb{R}^{d} \rightarrow \mathbb{R}^{d \times d}$ a matrix-valued function. We consider the system of stochastic differential equations

$$
\left\{\begin{array}{l}
d X_{t}^{i}=\sum_{j=1}^{d} A_{i j}\left(X_{t-}\right) d Z_{t}^{j}, \\
X_{0}^{i}=x_{0}^{i},
\end{array}\right.
$$

where $X_{t-}=\lim _{s \nearrow t} X_{s}$ is the left hand limit.

This system has been studied systematically in the case $\alpha_{1}=\alpha_{2}=\cdots=\alpha_{d}=\alpha \in(0,2)$ by Bass and Chen in the articles [3] and [4]. With the help of the martingale problem, Bass and Chen prove in [3] that for each $x_{0} \in \mathbb{R}^{d}$ there exists a unique weak solution $(X=$ $\left.\left(X_{t}^{1}, \ldots, X_{t}^{d}\right)_{t \geq 0}, \mathbb{P}^{x_{0}}\right)$ to (1.3). Furthermore the authors prove that the family $\left\{X, \mathbb{P}^{x}, x \in\right.$ $\left.\mathbb{R}^{d}\right\}$ forms a conservative strong Markov process on $\mathbb{R}^{d}$ whose semigroup maps bounded 
continuous functions to bounded continuous functions (see Theorem 1.1, [3]). Consequently it follows that

$$
\mathcal{L} f(x)=\sum_{j=1}^{d} \int_{\mathbb{R} \backslash\{0\}}\left(f\left(x+a_{j}(x) h\right)-f(x)-h \mathbb{1}_{\{|h| \leq 1\}} \nabla f(x) \cdot a_{j}(x)\right) \frac{c_{\alpha}}{|h|^{1+\alpha}} d h
$$

coincides on $C_{b}^{2}\left(\mathbb{R}^{d}\right)$ with the generator for any weak solution to (1.3), where $a_{j}(x)$ denotes the $\mathrm{j}^{\text {th }}$ column of the matrix $A(x)$. In [4] the authors prove Hölder regularity of harmonic functions with respect to $\mathcal{L}$ and give a counter example which shows that the Harnack inequality for harmonic functions is not satisfied.

In this paper we do not study unique solvability of (1.3) but prove an a-priori regularity estimate for harmonic functions if unique solutions to the system exist. The following assumptions will be needed throughout the paper.

Assumption (i) For every $x \in \mathbb{R}^{d}$ the matrix $A(x)$ is non-degenerate, that is $\operatorname{det}(A(x)) \neq 0$. (ii) The functions $x \mapsto A_{i j}(x)$ and $x \mapsto A_{i j}^{-1}(x)$ are continuous and bounded for all $1 \leq$ $i, j \leq d$ and $x \in \mathbb{R}^{d}$.

(iii) For any $x_{0} \in \mathbb{R}^{d}$, there exists a unique solution to the martingale problem for

$$
\mathcal{L} f(x)=\sum_{j=1}^{d} \int_{\mathbb{R} \backslash\{0\}}\left(f\left(x+a_{j}(x) h\right)-f(x)-h \mathbb{1}_{\{|h| \leq 1\}} \nabla f(x) \cdot a_{j}(x)\right) \frac{c_{\alpha_{j}}}{|h|^{1+\alpha_{j}}} d h
$$

started at $x_{0}$. The operator $\mathcal{L}$ coincides on $C_{b}^{2}\left(\mathbb{R}^{d}\right)$ with the generator for the weak solution to (1.3).

For a comprehensive introduction into the martingale problem we refer the reader to [16].

\section{Notation}

Let $A$ be the matrix-valued function from (1.3). Let $D$ be a Borel set. Throughout the paper $\varpi(D)$ denotes the modulus of continuity of $A$ and we write $\Lambda(D)$ for the upper bound of $A$ on $D$. We set $\alpha_{\min }:=\min \left\{\alpha_{1}, \ldots, \alpha_{d}\right\}$ and $\alpha_{\max }:=\max \left\{\alpha_{1}, \ldots, \alpha_{d}\right\}$. For $i \in \mathbb{N}$ we write $c_{i}$ for positive constants and additionally $c_{i}=c_{i}(\cdot)$ if we want to highlight all the quantities the constant depends on.

In order to deal with the anisotropy of the process we consider a corresponding scale of cubes.

Definition 1.1 Let $r \in(0,1]$ and $\alpha_{1}, \ldots, \alpha_{d} \in(0,2)$. For $k>0$, we define

$$
M_{r}^{k}(x):=\chi_{i=1}^{d}\left(x_{i}-\left(k r^{\alpha_{\max } / \alpha_{i}}\right), x_{i}+\left(k r^{\alpha_{\max } / \alpha_{i}}\right)\right) .
$$

For brevity we write $M_{r}(x)$ instead of $M_{r}^{1}(x)$.

Note that $M_{r}^{k}$ is increasing in $k$ and $r$. For $z \in \mathbb{R}^{d}$ and $r \in(0,1]$, the set $M_{r}(z)$ is a ball with radius $r$ and center $z$ in the metric space $\left(\mathbb{R}^{d}, d\right)$, where

$$
d(x, y)=\sup _{k \in\{1, \ldots, d\}}\left\{\left|x_{k}-y_{k}\right|^{\alpha_{k} / \alpha_{\max }} \mathbb{1}_{\left\{\left|x_{k}-y_{k}\right| \leq 1\right\}}(x, y)+\mathbb{1}_{\left\{\left|x_{k}-y_{k}\right|>1\right\}}(x, y)\right\} .
$$

This metric is useful for local considerations only, that is studies of balls with radii less or equal than one. The advantage of using these sets is the fact that they reflect the different jump intensities of the process $Z$ and compensate them in an appropriate way, see for instance Proposition 2.4. 
The purpose of this paper is to prove the following result.

Theorem 1.2 Let $r \in(0,1], s>0$ and $x_{0} \in \mathbb{R}^{d}$. Suppose $h$ is bounded in $\mathbb{R}^{d}$ and harmonic in $M_{r}^{1+s}\left(x_{0}\right)$ with respect to $X$. Then there exist $c_{1}=c_{1}\left(\Lambda\left(M_{r}^{1+s}\left(x_{0}\right)\right), \varpi\left(M_{r}^{1+s}\left(x_{0}\right)\right)\right)>0$ and $\beta=\beta\left(\Lambda\left(M_{r}^{1+s}\left(x_{0}\right)\right), \varpi\left(M_{r}^{1+s}\left(x_{0}\right)\right)\right)>0$, independent of $h$ and $r$, such that

$$
|h(x)-h(y)| \leq c_{1}\left(\frac{|x-y|}{r^{\alpha_{\max } / \alpha_{\min }}}\right)^{\beta} \sup _{\mathbb{R}^{d}}|h(z)| \text { for } x, y \in M_{r}\left(x_{0}\right) .
$$

We want to emphasize, that in the case $\alpha_{1}=\cdots=\alpha_{d}$ the set $M_{r}\left(x_{0}\right)$ reduces to a cube with radius $r$ and hence this result coincides with [4, Theorem 2.9], when one chooses cubes instead of balls.

Let us briefly discuss selected related results in the literature.

As previously mentioned, in [6] the authors study operators of the form (1.1) for coefficients $a: \mathbb{R}^{d} \times \mathbb{R}^{d} \rightarrow \mathbb{R}$ which are assumed to be symmetric in the second variable and satisfy $a(x, h) \asymp|h|^{-d-\alpha}$ for all $x, h \in \mathbb{R}^{d}$, where $\alpha \in(0,2)$. Using probabilistic techniques they prove a Harnack inequality and derive Hölder regularity estimates for bounded harmonic functions. The results of this work have been extended to more general kernels by several authors. For instance, in [5] the authors establish a Hölder estimate for harmonic functions to operators of the form (1.1), where they replace the jump measure $a(x, h) \mathrm{d} h$ by a family of measures $n(x, \mathrm{~d} h)$, which is not required to have a density with respect to the Lebesgue meaure. Furthermore, [32] extends the method of [6] to prove the Harnack inequality for more general classes of Markov processes. In [7] the authors construct and study the heat kernel a class of highly anisotropic integro-differential operators, where the Lévy measure does not have to be absolutely continuous with respect to the Lebesgue measure.

This article studies regularity for operators in non-divergence form given by (1.4). Hölder regularity results have intensively been studied for linear and nonlinear nonlocal equations governed by operators in non-divergence form. [31] provides a purely analytic proof of Hölder continuity for harmonic functions with respect to a class of integro differential equations given by (1.1), where no symmetry on the kernel $a$ is assumed. In [9], the authors study viscosity solutions to fully nonlinear integro-differential equations and prove a nonlocal version of the Aleksandrov-Bakelman-Pucci estimate, a Harnack inequality and a Hölder estimate. There are many more important results concerning Hölder estimates and Harnack inequalities for integro-differential equations in non-divergence form including [1,8,10,22,27] and [33]. Hölder regularity estimates have also been intensely studied for operators in divergence form. We would like to mention two works, where the corresponding jump intensities are similar to the ones we study in this article. In [12] and [13] the authors study nonlocal elliptic resp. parabolic equations for families of operators which can be of the form (1.2). They prove a weak Harnack inequality and Hölder regularity estimates for weak solutions to the corresponding equations.

Let us give a short survey to known results related to systems of stochastic differential equations given by (1.3). We first discuss some results in the case $\alpha_{1}=\cdots=\alpha_{d}$. In [3] the authors prove unique weak solvability for (1.3). [4] shows Hölder regularity estimates for bounded harmonic functions. Furthermore, in [26] the authors prove the strong Feller property for the corresponding semigroup for (1.3). Sharp lower bounds for the transition densities for the process $Z_{t}=\left(Z_{t}^{1}, \ldots, Z_{t}^{d}\right)$ are studied in [17] and sharp upper bounds in [21].

The existence of a unique solution to the martingale problem for (1.3) in the case of different orders of differentiability, i.e. $\alpha_{i} \neq \alpha_{j}$ for $i \neq j$, is shown in [11] under the additional assumption that the matrix $A$ is diagonal. [24] also studies the system (1.3) in the case of diagonal matrices $A$. The authors prove sharp two-sided estimates of the corresponding 
transition density $p^{A}(t, x, y)$ and prove Hölder and gradient estimates for the function $x \mapsto$ $p^{A}(t, x, y)$. In [18] the authors study the existence of densities for solutions (1.3) with Hölder continuous coefficients. They allow for a wide class of Lévy processes including the anisotropic processes $Z_{t}$ with different orders of differentiability. In [25] the authors study systems of the form (1.3) where $Z_{t}^{1}, \ldots, Z_{t}^{d}$ are independent one-dimensional Lévy processes with characteristic exponents $\psi_{1}, \ldots, \psi_{d}$. Under scaling conditions and regularity properties on the characteristic function they prove semigroup properties for solutions.

\section{Structure of the article}

This article is organized as follows. In Sect. 2 we provide definitions and auxiliary results. We constitute sufficient preparation and study the behavior of the solution to the system. In Sect. 3 we study the topological support of the solution to the martingale problem associated to the system of stochastic differential equations. The aim of this section is to prove a support theorem. Sect. 4 contains the proof of Theorem 1.2.

\section{Definitions and auxiliary results}

In this section we provide important definitions and prove auxiliary results associated to the solution of the system (1.3).

Let $A^{\tau}(x)$ denote the transpose of the matrix $A(x)$ and $\left(a_{j}^{\tau}(x)\right)^{-1}$ the $\mathrm{j}^{\text {th }}$ row of $\left(A^{\tau}(x)\right)^{-1}$. For a Borel set $D$, we denote the first entrance time of the process $X$ in $D$ by $T_{D}:=\inf \{t \geq$ $\left.0: X_{t} \in D\right\}$ and the first exit time of $X$ of $D$ by $\tau_{D}:=\inf \left\{t \geq 0: X_{t} \notin D\right\}$.

Let us first recall the definition of harmonicity with respect to a Markov process.

Definition 2.1 A bounded function $h: \mathbb{R}^{d} \rightarrow \mathbb{R}$ is called harmonic with respect to $X$ in a domain $D \subset \mathbb{R}^{d}$ if for every bounded open set $U$ with $U \Subset D$

$$
h\left(X_{t \wedge \tau_{U}}\right) \text { is a } \mathbb{P}^{x} \text {-martingale for every } x \in U .
$$

For $R=M_{s}(y)$ we use the notation $\widehat{R}=M_{s}^{3}(y)$. The next Proposition is a pure geometrical statement and not related to the system of stochastic differential equations. We skip the proof and refer the reader to [2, Proposition V.7.2], which can be easily adjusted to our case.

Proposition 2.2 Let $r \in(0,1], q \in(0,1)$ and $x_{0} \in \mathbb{R}^{d}$. If $A \subset M_{r}\left(x_{0}\right)$ and $|A|<q$, then there exists a set $D \subset M_{r}\left(x_{0}\right)$ such that

(1) $D$ is the union of rectangles $\widehat{R}_{i}$ such that the interiors of the $R_{i}$ are pairwise disjoint,

(2) $|A| \leq\left|D \cap M_{r}\left(x_{0}\right)\right|$ and

(3) for each $i,\left|A \cap R_{i}\right|>q\left|R_{i}\right|$.

Following the ideas of the proof of [6, Proposition 2.3], we next prove a Lévy system type formula.

Proposition 2.3 Suppose $D$ and $E$ are two Borel sets with $\operatorname{dist}(D, E)>0$. Then

$\sum_{s \leq t} \mathbb{1}_{\left\{X_{s-} \in D, X_{s} \in E\right\}}-\int_{0}^{t} \mathbb{1}_{D}\left(X_{s}\right) \int_{E} \sum_{k=1}^{d}\left(\frac{\left|\left(a_{k}^{\tau}\left(X_{s}\right)\right)^{-1}\right|^{1+\alpha_{k}} c_{\alpha_{k}}}{\left|h_{k}-X_{s}^{k}\right|^{1+\alpha_{k}}} d h_{k}\left(\prod_{j \neq k} \delta_{\left\{X_{s}^{j}\right\}}\left(d h_{j}\right)\right)\right) d s$

is a $\mathbb{P}^{x}$-martingale for each $x$. 
Proof Let $f \in C_{b}^{2}\left(\mathbb{R}^{d}\right)$ with $f=0$ on $D$ and $f=1$ on $E$. Moreover set

$$
M_{t}^{f}:=f\left(X_{t}\right)-f\left(X_{0}\right)-\int_{0}^{t} \mathcal{L} f\left(X_{s}\right) d s .
$$

By Assumption (iii) for each $x \in \mathbb{R}^{d}$ the probability measure $\mathbb{P}^{x}$ is a solution to the martingale problem for $\mathcal{L}$. Since the stochastic integral with respect to a martingale is itself a martingale,

$$
\int_{0}^{t} \mathbb{1}_{D}\left(X_{s-}\right) d M_{s}^{f}
$$

is a $\mathbb{P}^{x}$-martingale. Rewriting $f\left(X_{t}\right)-f\left(X_{0}\right)=\sum_{s \leq t}\left(f\left(X_{s}\right)-f\left(X_{s-}\right)\right)$ leads to

$$
\sum_{s \leq t}\left(\mathbb{1}_{D}\left(X_{s-}\right)\left(f\left(X_{s}\right)-f\left(X_{s-}\right)\right)\right)-\int_{0}^{t} \mathbb{1}_{D}\left(X_{s-}\right) \mathcal{L} f\left(X_{s}\right) d s
$$

is a $\mathbb{P}^{x}$-martingale. Since $X_{s} \neq X_{s-}$ for only countably many values of $s$,

$$
\sum_{s \leq t}\left(\mathbb{1}_{D}\left(X_{s-}\right)\left(f\left(X_{s}\right)-f\left(X_{s-}\right)\right)\right)-\int_{0}^{t} \mathbb{1}_{D}\left(X_{S}\right) \mathcal{L} f\left(X_{s}\right) d s
$$

is also a $\mathbb{P}^{x}$-martingale. Let $w=\left(w_{1}, \ldots, w_{d}\right)$ and $u=\left(u_{1}, \ldots, u_{d}\right)$. By definition of $f$, for $x \in D$ we have $f(x)=0$ and $\nabla f(x)=0$. Hence

$$
\begin{aligned}
\mathcal{L} f(x) & =\sum_{k=1}^{d} \int_{\mathbb{R} \backslash\{0\}} f\left(x+a_{k}(x) h\right) \frac{c_{\alpha_{k}}}{|h|^{1+\alpha_{k}}} d h \\
& =\sum_{k=1}^{d} \int_{\mathbb{R}^{d} \backslash\{0\}}\left(f\left(x+A^{\tau}(x) w\right) \frac{c_{\alpha_{k}}}{|w|^{1+\alpha_{k}}}\left(\prod_{j \neq k} \delta_{\{0\}}\left(d w_{j}\right)\right)\right) d w_{k} \\
& =\sum_{k=1}^{d} \int_{\mathbb{R}^{d} \backslash\{0\}} f(u) \frac{\left|\left(a_{k}^{\tau}(x)\right)^{-1}\right|^{1+\alpha_{k}} c_{\alpha_{k}}}{|u-x|^{1+\alpha_{k}}}\left(\prod_{j \neq k} \delta_{\left\{x_{j}\right\}}\left(d u_{j}\right)\right) d u_{k} .
\end{aligned}
$$

Note, that $c_{\alpha_{k}} /|h|^{1+\alpha_{k}}$ is integrable over $h$ in the complement of any neighborhood of the origin for any $k \in\{1, \ldots, d\}$. Since $D$ and $E$ have a positive distance from each other, the sum in (2.1) is finite. Hence

$$
\begin{aligned}
& \sum_{s \leq t}\left(\mathbb{1}_{D}\left(X_{s-}\right)\left(\mathbb{1}_{E}\left(X_{s}\right)-\mathbb{1}_{E}\left(X_{s}\right)\right)\right) \\
& -\int_{0}^{t} \mathbb{1}_{D}\left(X_{S}\right) \int_{E} \sum_{k=1}^{d}\left(\frac{\left|\left(a_{j}^{\tau}\left(X_{s}\right)\right)^{-1}\right|^{1+\alpha_{j}} c_{\alpha_{k}}}{\left|h_{k}-X_{s}^{k}\right|^{1+\alpha_{k}}} d h_{k}\left(\prod_{j \neq k} \delta_{\left\{X_{s}^{j}\right\}}\left(d h_{j}\right)\right)\right) d s
\end{aligned}
$$

is a $\mathbb{P}^{x}$-martingale, which is equivalent to our assertion.

The next Proposition gives the behavior of the expected first exit time of the solution to (1.3) out of the set $M_{r}(\cdot)$. This Proposition highlights the advantage of $M_{r}(\cdot)$ and shows that the scaling of the cube in the different directions with respect to the jump intensity compensates the different jump intensities in the different directions. 
Proposition 2.4 Let $x \in \mathbb{R}^{d}$ and $r \in(0,1]$. Then there exists a constant $c_{1}=$ $c_{1}\left(\Lambda\left(M_{r}(x)\right), d\right)>0$ such that for all $z \in M_{r}(x)$

$$
\mathbb{E}^{z}\left[\tau_{M_{r}(x)}\right] \leq c_{1} r^{\alpha_{\max }} .
$$

Proof First note

$$
\begin{aligned}
\mathbb{E}^{z}\left[\tau_{M_{r}(y)}\right] & =\mathbb{E}^{z}\left[\min _{1 \leq i \leq d} \inf \left\{t \geq 0: X_{t}^{i} \notin\left(y_{i}-r^{\left(\alpha_{\max } / \alpha_{i}\right)}, y_{i}-r^{\left(\alpha_{\max } / \alpha_{i}\right)}\right)\right\}\right] \\
& \leq \frac{1}{d} \sum_{i=1}^{d} \mathbb{E}^{z}\left[\inf \left\{t \geq 0: X_{t}^{i} \notin\left(y_{i}-r^{\left(\alpha_{\max } / \alpha_{i}\right)}, y_{i}-r^{\left(\alpha_{\max } / \alpha_{i}\right)}\right)\right\}\right] \\
& =: \frac{1}{d} \sum_{i=1}^{d} \mathbb{E}^{z}\left[\Upsilon_{i}\right] .
\end{aligned}
$$

Let $j \in\{1, \ldots, d\}$ be fixed but arbitrary. The aim is to show that there exists $c_{2}>0$ such that

$$
\mathbb{E}^{z}\left(\Upsilon_{j}\right) \leq c_{2} r^{\alpha_{\max }}
$$

Since we reduced the problem to a one-dimensional one, we may suppose by scaling $r=1$. Let

$$
\kappa:=\inf \left\{\left|A(x) e_{j}\right|: x \in \overline{M_{1}(x)}\right\} .
$$

By Assumption (i), we have $\kappa>0$. There exists a $c_{3} \in(0,1)$ with

$$
\mathbb{P}^{z}\left(\exists s \in[0,1]: \Delta Z_{s}^{j} \in \mathbb{R} \backslash[-3 / \kappa, 3 / \kappa]\right) \geq c_{3} .
$$

The independence of the one-dimensional processes implies that with probability zero at least two of the $Z^{i}$,s make a jump at the same time. This leads to

$$
\mathbb{P}^{z}\left(\exists s \in[0,1]: \Delta Z_{s}^{j}>\frac{3}{\kappa} \text { and } \Delta Z_{s}^{i}=0 \text { for } i \in\{1, \ldots, d\} \backslash\{j\}\right) \geq c_{3} .
$$

Our aim is to show that the probability of the process $X$ for leaving $M_{1}(x)$ in the $\mathrm{j}^{\text {th }}$ coordinate after time $m$ is bounded in the following way

$$
\mathbb{P}^{z}\left(\Upsilon_{j}>m\right) \leq\left(1-k_{j}\right)^{m} \text { for all } m \in \mathbb{N} \text {. }
$$

Suppose there exists $s \in[0,1]$ such that $\Delta Z_{s}^{j}>\frac{3}{\kappa}, \Delta Z_{s}^{i}=0$ for $i \in\{1, \ldots, d\} \backslash\{j\}$, and $X_{s-} \in M_{1}(x)$. Then

$$
\left|\Delta X_{s}^{j}\right|=\left|\Delta Z_{s}^{j}\right|\left|A\left(X_{s-}\right)\left(e_{j}\right)\right|>3 .
$$

Note, that we leave $M_{1}(x)$ by this jump. By (2.4)

$$
\mathbb{P}^{z}\left(\Upsilon_{j} \leq 1\right) \geq c_{3} \Leftrightarrow \mathbb{P}^{z}\left(\Upsilon_{j}>1\right) \leq\left(1-c_{3}\right) \text {. }
$$

Let $\left\{\theta_{t}: t \geq 0\right\}$ denote the shift operators for $X$. Now assume $\mathbb{P}^{z}\left(\Upsilon_{j}>m\right) \leq\left(1-c_{3}\right)^{m}$. By the Markov property

$$
\begin{aligned}
\mathbb{P}^{z}\left(\Upsilon_{j}>m+1\right) & \leq \mathbb{P}^{z}\left(\Upsilon_{j}>m ; \Upsilon_{j} \circ \theta_{m}>1\right) \\
& =\mathbb{E}^{z}\left[\mathbb{P}^{X}\left(\Upsilon_{j}>1\right) ; \Upsilon_{j}>m\right] \\
& \leq\left(1-c_{3}\right) \mathbb{P}^{z}\left(\Upsilon_{j}>m\right) \\
& \leq\left(1-c_{3}\right)^{m+1} .
\end{aligned}
$$


Assertion (2.3) follows by

$$
\mathbb{E}^{x}\left[\Upsilon_{j}\right]=\int_{0}^{\infty} \mathbb{P}^{z}\left(\Upsilon_{j}>t\right) d t \leq \sum_{m=0}^{\infty} \mathbb{P}^{z}\left(\Upsilon_{j}>m\right) \leq \sum_{m=0}^{\infty}\left(1-c_{3}\right)^{m}=c_{2},
$$

where we used the fact that the sum on the right hand side is a geometric sum. Thus the assertion follows by (2.2) and (2.3).

We close this section by giving an estimate for leaving a rectangle with a comparatively big jump.

Proposition 2.5 Let $x \in \mathbb{R}^{d}, r \in(0,1]$ and $R \geq 2 r$. There exists a constant $c_{1}=$ $c_{1}\left(\Lambda\left(\mathbb{R}^{d}\right), d\right)>0$, such that for all $z \in M_{r}(x)$

$$
\mathbb{P}^{z}\left(X_{\tau_{M_{r}(x)}} \notin M_{R}(x)\right) \leq c_{1}\left(\frac{r}{R}\right)^{\alpha_{\max }} .
$$

Proof Let

$$
C_{j}:=\mathbb{R} \backslash\left[x_{j}-R^{\alpha_{\max } / \alpha_{j}}, x_{j}+R^{\alpha_{\max } / \alpha_{j}}\right]
$$

and for $1 \leq j \leq d$ let $k_{j}=\sup _{x \in \mathbb{R}}\left|\left(a_{j}^{\tau}(x)\right)^{-1}\right| c_{\alpha_{j}}$. By Proposition 2.3 and optional stopping we get for $c_{2}=\sum_{j=1}^{d}\left(\left(2 k_{j} 2_{\max }^{\alpha}\right) / c_{\alpha_{j}}\right) \leq 8 d \sup _{x \in \mathbb{R}}\left|\left(a_{j}^{\tau}(x)\right)^{-1}\right|$

$$
\begin{aligned}
\mathbb{P}^{z}\left(X_{t \wedge \tau_{M_{r}(x)}} \notin M_{R}(x)\right) & =\mathbb{E}^{z}\left[\int_{0}^{t \wedge \tau_{M_{r}(x)}} \int_{M_{R}(x)^{c}} \sum_{j=1}^{d} \frac{\left|\left(a_{j}^{\tau}\left(X_{s}\right)\right)^{-1}\right| c_{\alpha_{j}}}{\left|h_{j}-X_{s}^{j}\right|^{1+\alpha_{j}}}\left(\prod_{i \neq j} \delta_{\left\{X_{s}^{i}\right\}}\left(d h_{i}\right)\right) d h_{j} d s\right] \\
& \leq \mathbb{E}^{z}\left[\int_{0}^{t \wedge \tau_{M_{r}(x)}} \sum_{j=1}^{d} \int_{C_{j}} \frac{k_{j}}{\left|h_{j}-X_{s}^{j}\right|^{1+\alpha_{j}}} d h_{j} d s\right] \\
& \leq \mathbb{E}^{z}\left[\int_{0}^{t \wedge \tau_{M_{r}(x)}} \sum_{j=1}^{d} \int_{C_{j}} \frac{k_{j}}{\left|h_{j}-\left(x_{j}+r^{\alpha_{\max } / \alpha_{j}}\right)\right|^{1+\alpha_{j}}} d h_{j} d s\right] \\
& =\mathbb{E}^{z}\left[t \wedge \tau_{\left.M_{r}(x)\right)}\right] \sum_{j=1}^{d} \frac{2 k_{j}}{\alpha_{j}\left(R^{\alpha_{\max } / \alpha_{j}}-r^{\alpha_{\max } / \alpha_{j}}\right)^{\alpha_{j}}} \\
& \leq \mathbb{E}^{z}\left[t \wedge \tau_{\left.M_{r}(x)\right)} \sum_{j=1}^{d} \frac{2 k_{j}}{\alpha_{j}\left((R / 2)^{\alpha_{\max } / \alpha_{j}}\right)^{\alpha_{j}}}=\frac{c_{2}}{R^{\alpha_{\max }}} \mathbb{E}^{z}\left[t \wedge \tau_{\left.\left.M_{r}(x)\right)\right] .}\right.\right.
\end{aligned}
$$

Using the monotone convergence on the right and dominated convergence on the left, we have for $t \rightarrow \infty$

$$
\mathbb{P}^{z}\left(X_{t \wedge \tau_{M_{r}(x)}} \notin M_{R}(x)\right) \leq \frac{c_{2}}{R^{\alpha_{\max }}} \mathbb{E}^{z}\left(\tau_{M_{r}(x)}\right) \leq c_{2} c_{3}\left(\frac{r}{R}\right)^{\alpha_{\max }},
$$

where $c_{3}$ is the constant showing up in the estimate $\mathbb{E}^{z}\left(\tau_{M_{r}(x)}\right) \leq c_{3} r^{\alpha}$ of Proposition 2.4.

\section{The support theorem}

In this section we prove the main ingredient for the proof of the Hölder regularity estimate for harmonic functions. The so-called support theorem states that sets of positive Lebesgue measure are hit with positive probability. 
This theorem was first proved in [23] for the diffusion case. In the article [4], Bass and Chen prove the support theorem in the context of pure jump processes with singular and anisotropic kernels. They consider the system (1.3) in the case $\alpha_{i}=\alpha$ for all $i \in\{1, \ldots, d\}$ and use the technique by Krylov and Safonov to prove Hölder regularity with the help of the support theorem.

The idea we use to prove the support theorem is similar in spirit to the one in [4].

The following Lemma is a statement about the topological support of the law of the stopped process. It gives the existence of a bounded stopping time $T$ such that with positive probability the stopped process stays in a small ball around its starting point up to time $T$, makes a jump along the $\mathrm{k}^{\text {th }}$ coordinate axis and stays afterwards in a small ball.

Lemma 3.1 Let $r \in(0,1], x_{0} \in \mathbb{R}^{d}, k \in\{1, \ldots, d\}, v_{k}=A\left(x_{0}\right) e_{k}, \gamma \in\left(0, r^{\alpha_{\max } / \alpha_{\min }}\right), t_{0}>$ 0 and $\xi \in\left[-r^{\alpha_{\max } / \alpha_{\min }}, r^{\alpha_{\max } / \alpha_{\min }}\right]$. There exists a constant $c_{1}>0=c_{1}\left(\gamma, t_{0}, \xi, r\right.$, $\left.\left.\Lambda\left(M_{r}^{2}\left(x_{0}\right)\right)\right), \varpi\left(M_{r}^{2}\left(x_{0}\right)\right)\right)>0$ and a stopping time $T \leq t_{0}$, such that

$$
\mathbb{P}^{x_{0}}\left(\sup _{s<T}\left|X_{s}-x_{0}\right|<\gamma \text { and } \sup _{T \leq s \leq t_{0}}\left|X_{s}-\left(x_{0}+\xi v_{k}\right)\right|<\gamma\right) \geq c_{1} .
$$

Proof Let

$$
\|A\|_{\infty}:=1 \vee\left(\sum_{i, j=1}^{d} \sup _{x \in M_{r}^{2}\left(x_{0}\right)}\left|A_{i j}(x)\right|\right) .
$$

We assume $\xi \in\left[0, r^{\alpha_{\max } / \alpha_{\min }}\right]$. The case $\xi \in\left[-r^{\alpha_{\max } / \alpha_{\min }}, 0\right]$ can be proven similar. Let us first suppose $\xi \geq \gamma /\left(3\|A\|_{\infty}\right)$ and let $\beta \in(0, \xi)$, which will be chosen later. We decompose the process $Z_{t}^{i}$ in the following way:

$$
\widetilde{Z}_{t}^{i}=\sum_{s \leq t} \Delta Z_{s}^{i} \mathbb{1}_{\left\{\left|\Delta Z_{s}^{i}\right|>\beta\right\}}, \quad \bar{Z}_{t}^{i}=Z_{t}^{i}-\widetilde{Z}_{t}^{i}
$$

Let $\left(\bar{X}_{t}\right)_{t \geq 0}$ be the solution to

$$
d \bar{X}_{t}^{i}=\sum_{j=1}^{d} A_{i j}\left(\bar{X}_{t-}\right) d \bar{Z}_{t}^{j}, X_{0}^{i}=x_{0}^{i} .
$$

The continuity of $A$ allows us to find a $\delta<\gamma /\left(6\|A\|_{\infty}\right)$, such that

$$
\sup _{i, j} \sup _{\left|x-x_{0}\right|<\delta}\left|A_{i j}(x)-A_{i j}\left(x_{0}\right)\right|<\frac{\gamma}{12 d} .
$$

Consider

$$
\begin{aligned}
C= & \left\{\sup _{s \leq t_{0}}\left|\bar{X}_{s}-\bar{X}_{0}\right| \leq \delta\right\}, \\
D= & \left\{\widetilde{Z}^{k} \text { has precisely one jump before time } t_{0} \text { with jump size in }[\xi, \xi+\delta],\right. \\
& \left.\Delta \widetilde{Z}_{s}^{j}=0 \text { for all } s \leq t_{0} \text { and all } j \neq k\right\}, \\
E= & \left\{\widetilde{Z}_{s}^{i}=0 \text { for all } s \leq t_{0} \text { and } i=1, \ldots, d\right\} .
\end{aligned}
$$


Since $A$ is bounded, we can find $c_{2}>0$, such that

$$
\left[\bar{X}^{i}, \bar{X}^{i}\right]_{t} \leq c_{2} \sum_{j=1}^{d}\left[\bar{Z}^{j}, \bar{Z}^{j}\right]_{t}
$$

Note, that $\beta \in(0, \xi) \subset\left(0, r^{\alpha_{\max } / \alpha_{\min }}\right) \subset(0,1)$. Therefore, we get

$$
\mathbb{E}^{x_{0}}\left[\bar{X}^{i}, \bar{X}^{i}\right]_{t} \leq c_{2} \sum_{j=1}^{d} \mathbb{E}^{x_{0}}\left[\bar{Z}^{j}, \bar{Z}^{j}\right]_{t}=c_{2} \sum_{j=1}^{d} \int_{0}^{t}\left(\int_{-\beta}^{\beta} \frac{c_{\alpha_{j}} h^{2}}{|h|^{1+\alpha_{j}}} d h\right) d t \leq c_{3} t d \beta^{2-\alpha_{\max }} .
$$

By Tschebyscheff's inequality and Doob's inequality, we get

$$
\begin{aligned}
\mathbb{P}^{x_{0}}\left[\sup _{s \leq t_{0}}\left|\bar{X}_{s}^{i}-\bar{X}_{0}^{i}\right|>\delta\right] \leq \frac{1}{\delta^{2}} \mathbb{E}^{x_{0}}\left[\sup _{s \leq t_{0}}\left(\bar{X}_{s}^{i}-\bar{X}_{0}^{i}\right)^{2}\right] & \leq \frac{1}{\delta^{2}} 4 \mathbb{E}^{x_{0}}\left[\left(\bar{X}_{t_{0}}^{i}-\bar{X}_{0}^{i}\right)^{2}\right] \\
& \leq \frac{c_{4} t_{0} d \beta^{2-\alpha_{\max }}}{\delta^{2}}
\end{aligned}
$$

Choose $\beta \in(0, \xi)$ such that

$$
c_{5} t_{0} \beta^{2-\alpha_{\max }} \leq \frac{\delta^{2}}{2 d}
$$

holds. Then by (3.3), we get

$$
\mathbb{P}^{x_{0}}(C)=1-\mathbb{P}^{x_{0}}\left(\sup _{s \leq t_{0}}\left|\bar{X}_{s}^{i}-\bar{X}_{0}^{i}\right|>\delta\right) \geq \frac{1}{2} .
$$

For $\widetilde{Z}^{k}$ to have a single jump before time $t_{0}$, and for that jump's size to be in the interval $[\xi, \xi+\delta]$, then up to time $t_{0} \widetilde{Z}_{t}^{k}$ must have

(i) no negative jumps,

(ii) no jumps whose size lies in $[\beta, \xi)$,

(iii) no jumps whose size lies in $(\xi+\delta, \infty)$,

(iv) precisely one jump whose size lies in the interval $[\xi, \xi+\delta]$.

We can use the fact, that $\widetilde{Z}^{k}$ is a compound Poisson process and use the knowledge about Poisson random measures. The events descriped in (i)-(iv) are the probabilities that Poisson random variables $P_{1}, P_{2} . P_{3}$ and $P_{4}$ of parameters $\lambda_{1}=c_{6} t_{0} \beta^{-\alpha_{k}}, \lambda_{2}=c_{6} t_{0}\left(\beta^{-\alpha_{k}}-\xi^{-\alpha_{k}}\right)$, $\lambda_{3}=c_{6} t_{0}(\xi+\delta)^{-\alpha_{k}}$, and $\lambda_{4}=c_{6} t_{0}\left(\xi^{-\alpha_{k}}-(\xi+\delta)^{-\alpha_{k}}\right)$, respectively, take the values $0,0,0$, and 1 , respectively.

So there exists a constant $c_{7}=c_{7}\left(\alpha_{k}, t_{0}, \delta, \xi, \beta\right)>0$ such that

$$
\mathbb{P}^{x_{0}}\left(\widetilde{Z}^{k} \text { has a single jump before time } t_{0} \text {, and its size is in }[\xi, \xi+\delta]\right) \geq c_{7} \text {. }
$$

For all $j \neq k$, the probability that $\widetilde{Z}^{j}$ does not have a jump before time $t_{0}$, is the probability that a Poisson random variable with parameter $2 c_{6} t_{0} \beta^{-\alpha_{j}}$ is equal to 0 . Using the indepence of $\widetilde{Z}^{j}$ for $j=1, \ldots, d$, we can find a $c_{8}>0$ such that

$$
\mathbb{P}^{x_{0}}\left(\Delta \widetilde{Z}_{s}^{j}=0 \text { for all } s \leq t_{0} \text { and all } j \neq k\right) \geq c_{8} .
$$

Thus we obtain

$$
\mathbb{P}^{x_{0}}(D) \geq c_{9}
$$


for a $c_{9}=c_{9}\left(\alpha_{1}, \ldots, \alpha_{d}, t_{0}, \delta, \xi, \beta\right)>0$. Furthermore the $\bar{Z}^{i}$ 's are independent of the $\widetilde{Z}^{j}$ 's for all $i, j \in\{1, \ldots, d\}$, so $C$ and $D$ are independent and we obtain

$$
\mathbb{P}^{x_{0}}(C \cap D) \geq c_{9} / 2 \text {. }
$$

Similary we obtain

$$
\mathbb{P}^{x_{0}}(E) \geq c_{10} \text { and } \mathbb{P}^{x_{0}}(C \cap E) \geq c_{11} .
$$

Let $T$ be the time, when $\widetilde{Z}^{k}$ jumps the first time, i.e. $Z^{k}$ makes a jump greater then $\beta$. Then $Z_{s-}=\bar{Z}_{s-}$ for all $s \leq T$ and hence $X_{s-}=\bar{X}_{s-}$ for all $s \leq T$. So up to time $T, X_{s}$ does not move away more than $\delta$ away from its starting point. Note $\Delta X_{T}=A\left(X_{T-}\right) \Delta Z_{T}$. By (3.2), we obtain on $C \cap D$

$$
\begin{aligned}
\left|X_{T}-\left(x_{0}+\xi v_{k}\right)\right| & \left.\left.\leq\left|X_{T-}-x_{0}\right|+\mid \Delta X_{T}-\xi A\left(x_{0}\right) e_{k}\right)\right) \mid \\
& \left.=\left|X_{T-}-x_{0}\right|+\mid A\left(X_{T-}\right) \Delta Z_{T}-\xi A\left(x_{0}\right) e_{k}\right) \mid \\
& \leq\left|X_{T-}-x_{0}\right|+\xi\left|\left(A\left(X_{T-}\right)-A\left(x_{0}\right)\right) e_{k}\right|+\left|A\left(X_{T-}\right)\left(\Delta Z_{T}-\xi e_{k}\right)\right| \\
& \leq \delta+\frac{\xi d \gamma}{12 d}+\delta\|A\|_{\infty} \leq \frac{\gamma}{6}\left(\frac{1}{\|A\|_{\infty}}+\frac{\gamma}{2}+1\right) \leq \frac{\gamma}{2} .
\end{aligned}
$$

Appling the strong Markov property at time $T$, we get by (3.5)

$$
\mathbb{P}^{x_{0}}\left(\sup _{T \leq s \leq T+t_{0}}\left|X_{s}-X_{T}\right|<\delta\right) \geq \mathbb{P}^{X_{T}}(C \cap E) \geq c_{11} .
$$

Note, that $\left|X_{T}-\left(x_{0}+\xi v_{k}\right)\right|<\gamma / 2$ and $\left|X_{s}-X_{T}\right|<\delta$ for all $T \leq s \leq t_{0}$ imply $\left|X_{s}-\left(x_{0}+\xi v_{k}\right)\right|<\gamma$.

All in all we get by the strong Markov property

$$
\mathbb{P}^{x_{0}}\left(\sup _{s<T}\left|X_{s}-x_{0}\right|<\gamma \text { and } \sup _{T \leq s \leq t_{0}}\left|X_{s}-\left(x_{0}+\xi v_{k}\right)\right|<\gamma\right) \geq \frac{c_{9} c_{11}}{2},
$$

which proves the assertion.

Now suppose $\xi<\gamma /\left(3\|A\|_{\infty}\right)$. Then $\left|x_{0}-\left(x_{0}+\xi v_{k}\right)\right|<\gamma / 3$. We can choose $T \equiv 0$ and by (3.5) we get:

$$
\mathbb{P}^{x_{0}}\left(\sup _{s \geq t_{0}}\left|X_{s}-x_{0}\right|<\delta\right) \geq c_{11}
$$

which finishes the proof.

We need two simple geometrical facts from the field of linear algebra, whose proofs can be found in [4] (Lemmas 2.4 and 2.5).

Lemma 3.2 Suppose $u, v$ are two vectors in $\mathbb{R}^{d}, \eta \in(0,1)$, and $p$ is the projection of $v$ onto u. If $|p| \geq \eta|v|$, then

$$
|v-p| \leq \sqrt{1-\eta^{2}}|v|
$$

Lemma 3.3 Let $v$ be a vector in $\mathbb{R}^{d}, u_{k}=A e_{k}$, and $p_{k}$ the projection of $v$ onto $u_{k}$ for $k=1, \ldots, d$. Then there exists $\rho=\rho\left(\Lambda\left(\mathbb{R}^{d}\right)\right) \in(0,1)$, such that for some $k$,

$$
\left|v-p_{k}\right| \leq \rho|v| \text {. }
$$


For a given time $t_{1}>0$ the following lemma shows that solutions stay with positive probability in an $\varepsilon$-tube around a given line segment on $\left[0, t_{1}\right]$. The case of $\alpha_{1}=\cdots=\alpha_{d}$ was considered in [4]. We follow their technique.

Lemma 3.4 Let $r \in(0,1], x_{0} \in \mathbb{R}^{d}, t_{1}>0, \varepsilon \in\left(0, r^{\alpha_{\max } / \alpha_{\min }}\right), \xi \in(0, \varepsilon / 4)$ and $\gamma>0$. Moreover let $\psi:\left[0, t_{1}\right] \rightarrow \mathbb{R}^{d}$ be a line segment of length $\xi$ starting at $x_{0}$. Then there exists $\left.\left.c_{1}=c_{1}\left(\Lambda\left(M_{r}^{2}\left(x_{0}\right)\right)\right), \varpi\left(M_{r}^{2}\left(x_{0}\right)\right)\right), t_{1}, \varepsilon, \gamma\right)>0$, such that

$$
\mathbb{P}^{x_{0}}\left(\sup _{s \leq t_{1}}\left|X_{s}-\psi(s)\right|<\varepsilon \text { and }\left|X_{t_{1}}-\psi\left(t_{1}\right)\right|<\gamma\right) \geq c_{1} .
$$

Proof Note that $\varepsilon$ is chosen such that $B_{\varepsilon}\left(x_{0}\right) \subset M_{r}\left(x_{0}\right)$. Let $\rho \in(0,1)$ be such that the conclusion of Lemma 3.3 holds for all matrices $A=A(x)$ with $x \in M_{r}^{2}(x)$. Take $\gamma \in$ $(0, \xi \wedge \rho)$ such that $\widetilde{\rho}:=\gamma+\rho<1$ and $n \geq 2$ sufficiently large, such that $(\widetilde{\rho})^{n}<\gamma$. Let $v_{0}:=\psi\left(t_{1}\right)-\psi(0)=\psi\left(t_{1}\right)-x_{0}$, which has length $\xi$. By Lemma 3.2, there exists a $k_{0} \in\{1, \ldots, d\}$ such that if $p_{0}$ is the projection of $v_{0}$ onto $A\left(x_{0}\right) e_{k_{0}}$, then $\left|v_{0}-p_{0}\right| \leq \rho\left|v_{0}\right|$. Note, that $\left|p_{0}\right| \leq\left|v_{0}\right|=\xi$. By Lemma 3.1 there exists $c_{2}>0$ and a stopping time $T_{0} \leq t_{1} / n$ such that for

$$
D_{1}:=\left\{\sup _{s<T_{0}}\left|X_{s}-x_{0}\right|<\gamma^{n+1} \text { and } \sup _{T_{0} \leq s \leq t_{1} / n}\left|X_{s}-\left(x_{0}+p_{0}\right)\right|<\gamma^{n+1}\right\} .
$$

the estimate

$$
\mathbb{P}^{x_{0}}\left(D_{1}\right) \geq c_{2}
$$

holds. Since $\gamma<1$ and $\gamma^{n} \leq \gamma$ for all $n \in \mathbb{N}$, we have for $T_{0} \leq s \leq t_{1} / n$

$$
\begin{aligned}
\left|\psi\left(t_{1}\right)-X_{s}\right| & \leq\left|\psi\left(t_{1}\right)-\left(x_{0}+p_{0}\right)\right|+\left|\left(x_{0}+p_{0}\right)-X_{s}\right| \\
& \leq\left|v_{0}-p_{0}\right|+\gamma^{n+1}=\rho \xi+\gamma^{n+1} \leq \widetilde{\rho} \xi
\end{aligned}
$$

on $D_{1}$. Taking $s=t_{1} / n$, we have

$$
\left|\psi\left(t_{1}\right)-X_{t_{1} / n}\right| \leq \widetilde{\rho} \xi
$$

Since $\tilde{\rho}<1$ and $\left|\psi\left(t_{1}\right)-x_{0}\right|=\left|v_{0}\right|=\xi$, then (3.6) shows that on $D_{1}$

$$
X_{s} \in B\left(x_{0}, 2 \xi\right) \subset B\left(x_{0}, \varepsilon / 2\right) \text { if } T_{0} \leq s \leq t_{1} / n .
$$

If $0 \leq s<T_{0}$, then $\left|X_{s}-x_{0}\right|<\gamma^{n+1}<\xi$, and so we have on $D_{1}$

$$
\left\{X_{s}, s \in\left[0, t_{1} / n\right]\right\} \subset B\left(x_{0}, 2 \xi\right) \subset B\left(x_{0}, \varepsilon / 2\right) .
$$

Now let $v_{1}:=\psi\left(t_{1}\right)-X_{t_{1} / n}$. When $X_{t_{1} / n} \in B\left(x_{0}, \varepsilon / 2\right)$, then by Lemma 3.3, there exists $k_{1} \in\{1, \ldots, d\}$ such that if $p_{1}$ is the projection of $v_{1}$ onto $A\left(X_{t_{1} / n}\right) e_{k_{1}}$, then $\left|v_{1}-p_{1}\right| \leq \rho\left|v_{1}\right|$. Let $T_{1} \in\left[t_{1} / n, 2 t_{1} / n\right]$ be a stopping time, determined by Lemma 3.1, and

$$
D_{2}:=\left\{\sup _{t_{1} / n \leq s<T_{1}}\left|X_{s}-X_{t_{1} / n}\right|<\gamma^{n+1} \text { and } \sup _{T_{1} \leq s \leq 2 t_{1} / n}\left|X_{s}-\left(X_{t_{1} / n}+p_{1}\right)\right|<\gamma^{n+1}\right\} .
$$

By the Markov property at the time $t_{1} / n$ and Lemma 3.1, there exists the same $c_{2}>0$ such that

$$
\mathbb{P}^{x_{0}}\left(D_{2} \mid \mathcal{F}_{t_{1} / n}\right) \geq c_{2}
$$


on the event $\left\{X_{t_{1} / n} \in B\left(x_{0}, \varepsilon / 2\right)\right\}$ and hence especially on $D_{1}$. So

$$
\mathbb{P}^{x_{0}}\left(D_{1} \cap D_{2}\right) \geq c_{2} \mathbb{P}^{x_{0}}\left(D_{1}\right) \geq c_{2}^{2} .
$$

Let $s \in\left[T_{1}, 2 t_{1} / n\right]$. Then on $D_{1} \cap D_{2}$

$$
\begin{aligned}
\left|\psi\left(t_{1}\right)-X_{s}\right| & \leq\left|\psi\left(t_{1}\right)-\left(X_{t_{1} / n}+p_{1}\right)\right|+\left|\left(X_{t_{1} / n}+p_{1}\right)-X_{s}\right| \\
& \leq \rho\left|v_{1}\right|+\gamma^{n+1} \leq \rho \widetilde{\rho} \xi+\gamma^{n+1} \leq(\widetilde{\rho})^{2} \xi+\gamma^{n+1} \\
& \leq \widetilde{\rho}^{2} \xi .
\end{aligned}
$$

In particular, by choosing $s=2 t_{1} / n$, we get on $D_{1} \cap D_{2}$

$$
\left|\psi\left(t_{1}\right)-X_{2 t_{1} / n}\right| \leq(\widetilde{\rho})^{2} \xi .
$$

On $D_{1} \cap D_{2}$, we have for $s \in\left[T_{1}, 2 t_{1} / n\right]:\left|\psi\left(t_{1}\right)-X_{s}\right|<\xi$ and $\left|\psi\left(t_{1}\right)-x_{0}\right|=\xi$, which implies

$$
X_{s} \in B\left(x_{0}, 2 \xi\right) \subset B\left(x_{0}, \varepsilon / 2\right) \quad \text { on } D_{1} \cap D_{2} .
$$

In particular,

$$
\left|X_{2 t_{1} / n}-x_{0}\right|<2 \xi \quad \text { on } D_{1} \cap D_{2} .
$$

If $s \in\left[t_{1} / n, T_{1}\right]$, then $\left|X_{s}-X_{t_{1} / n}\right|<\xi$ and $\left|X_{t_{1} / n}-x_{0}\right|<2 \xi$ on $D_{1} \cap D_{2}$, which yields to

$$
X_{s} \in B\left(x_{0}, 3 \xi\right) \subset B\left(x_{0}, 3 \varepsilon / 4\right) \quad \text { on } D_{1} \cap D_{2} .
$$

Let $v_{2}:=\psi\left(t_{1}\right)-X_{2 t_{1} / n}$, and proceed as above to get events $D_{3}, \ldots, D_{k}$ for $k \leq n$. At the $\mathrm{k}^{\text {th }}$ stage

$$
\mathbb{P}^{x_{0}}\left(D_{k} \mid F_{(k-1) t_{1} / n}\right) \geq c_{2} \quad \text { and so } \quad \mathbb{P}^{x_{0}}\left(\bigcap_{j=1}^{k} D_{j}\right) \geq c_{2}^{k} .
$$

For $k t_{1} / n \leq T_{k} \leq s \leq(k+1) t_{1} / n$

$$
\left|\psi\left(t_{1}\right)-X_{s}\right| \leq(\widetilde{\rho})^{k+1} \xi<\xi
$$

on the event $\bigcap_{j=1}^{k} D_{j}$. Since $\left|\psi\left(t_{1}\right)-x_{0}\right|=\xi$.

$$
X_{s} \in B\left(x_{0}, 2 \xi\right) \subset B\left(x_{0}, \varepsilon / 2\right) \text { on } \bigcap_{j=1}^{k} D_{j} .
$$

If $k t_{1} / n \leq s<T_{k}$, we obtain at the kth stage

$$
\left|X_{k t_{1} / n}-x_{0}\right|<\varepsilon / 2 \text { on } \bigcap_{j=1}^{k} D_{j} .
$$

Thus

$$
\left|X_{s}-x_{0}\right| \leq\left|X_{s}-X_{k t_{1} / n}\right|+\left|X_{k t_{1} / n}-\psi\left(t_{1}\right)\right|+\left|\psi\left(t_{1}\right)-x_{0}\right| \leq \gamma^{n+1}+\xi+\xi<3 \xi,
$$

and therefore $X_{s} \in B\left(x_{0}, 3 \xi\right) \subset B\left(x_{0}, 3 \varepsilon / 4\right)$. We continue this procedure $n$ times to get events $D_{1}, \ldots, D_{n}$. On $\bigcap_{k=1}^{n} D_{k}$, we have

(1) $X_{s} \in B\left(x_{0}, 3 \xi\right)$ for $s \leq t_{1}$,

(2) $\left|X_{t_{1}}-\psi\left(t_{1}\right)\right|<(\widetilde{\rho})^{n} \xi<(\widetilde{\rho})^{n}<\gamma$, and 
(3) $\mathbb{P}^{x_{0}}\left(\bigcap_{j=1}^{n} D_{j}\right) \geq c_{2}^{n}$.

For $s \in\left[0, t_{1}\right]$,

$$
\left|X_{s}-\psi(s)\right| \leq\left|X_{s}-x_{0}\right|+\left|x_{0}-\psi(s)\right|<3 \xi+\xi<\frac{3}{4} \varepsilon+\frac{1}{4} \varepsilon=\varepsilon \quad \text { on } \bigcap_{j=1}^{n} D_{j} .
$$

Hence

$$
\mathbb{P}^{x_{0}}\left(\sup _{s \leq t_{1}}\left|X_{s}-\psi(s)\right|<\varepsilon \text { and }\left|X_{t_{1}}-\psi\left(t_{1}\right)\right|<\gamma\right) \geq \mathbb{P}^{x_{0}}\left(\bigcap_{j=1}^{n} D_{j}\right) \geq c_{2}^{n}=: c_{1} .
$$

We can now prove an important theorem, which will be the main ingredient in the proof of the Hölder regularity. It states that the solution to (1.3) stays with positive probability in a $\varepsilon$-tube around a given continuous function.

Theorem 3.5 Let $r \in(0,1], x_{0} \in \mathbb{R}^{d}, \varepsilon \in\left(0, r^{\alpha_{\max } / \alpha_{\min }}\right), t_{0}>0$ and $x_{0} \in \mathbb{R}^{d}$. Let $\varphi$ : $\left[0, t_{0}\right] \rightarrow \mathbb{R}^{d}$ be continuous with $\varphi(0)=x_{0}$ and the image of $\varphi$ contained in $M_{r}\left(x_{0}\right)$. Then there exists $c_{1}=c_{1}\left(\Lambda\left(M_{r}^{2}\left(x_{0}\right)\right), \varpi\left(M_{r}^{3}\left(x_{0}\right)\right), \varphi, \varepsilon, t_{0}\right)>0$ such that

$$
\mathbb{P}^{x_{0}}\left(\sup _{s \leq t_{0}}\left|X_{s}-\varphi(s)\right|<\varepsilon\right)>c_{1} .
$$

Proof Let $\varepsilon>0$. We define

$$
U:=\left\{x \in \mathbb{R}^{d}: \exists s \in\left[0, t_{0}\right] \text { such that }|x-\varphi(s)|<\varepsilon / 2\right\}
$$

and approximate $\varphi$ within $U$ by a polygonal path. Hence we can assume that $\varphi$ is polygonal by changing $\varepsilon$ to $\varepsilon / 2$ in the assertion. We subdivide $\left[0, t_{0}\right]$ into $n$ subintervals of the same length for $n \geq 2$ such that

$$
r:=L\left(\varphi\left(\left(\frac{k t_{0}}{n}, \frac{(k+1) t_{0}}{n}\right)\right)\right)<\frac{\varepsilon}{4},
$$

where $L$ denotes the length of the line segment. Let

$$
D_{k}:=\left\{\sup _{(k-1) t_{0} / n \leq s \leq k t_{0} / n}\left|X_{s}-\varphi(s)\right|<\frac{\varepsilon}{2} \text { and }\left|X_{k t_{0} / n}-\varphi\left(k t_{0} / n\right)\right|<\frac{\varepsilon}{4 \sqrt{d}}\right\} .
$$

Using Lemma 3.4, there exists a constant $c_{2}>0$ such that

$$
\mathbb{P}^{x_{0}}\left(D_{1}\right) \geq c_{2} \text {. }
$$

By the strong Markov property at time $t_{0} / n$ we get

$$
\mathbb{P}^{x_{0}}\left(D_{2} \mid \mathcal{F}_{t_{0} / n}\right) \geq c_{2} \text {. }
$$

Using the Iteration as in the proof of Lemma 3.4, we get for all $k \in\{1, \ldots, d\}$

$$
\mathbb{P}^{x_{0}}\left(D_{k} \mid \mathcal{F}_{(k-1) t_{0} / n}\right) \geq c_{2} \text { and } \mathbb{P}^{x_{0}}\left(\bigcap_{k=1}^{n} D_{k}\right) \geq c_{2}^{n}
$$


Hence the assertion follows by

$$
\mathbb{P}^{x_{0}}\left(\sup _{s \leq t_{0}}\left|X_{s}-\varphi(s)\right|<\frac{\varepsilon}{2}\right) \geq \mathbb{P}^{x_{0}}\left(\bigcap_{k=1}^{n} D_{k}\right) \geq c_{2}^{n}=c_{1} .
$$

We state two corollaries, which follow immediately from Theorem 3.5.

Corollary 3.6 Let $r \in(0,1], \varepsilon \in\left(0, r^{\alpha_{\max } / \alpha_{\min }} / 4\right), k=1-\left(\varepsilon / r^{\alpha_{\max } / \alpha_{\min }}\right), \delta \in$ $\left(\varepsilon, r^{\alpha_{\max } / \alpha_{\min }} / 2\right)$ and $x_{0} \in \mathbb{R}^{d}$. Moreover let $Q:=M_{r}\left(x_{0}\right), Q^{\prime}:=M_{r}^{k}\left(x_{0}\right)$ and $y \in \mathbb{R}^{d}$ such that $R:=M_{r}^{\delta}(y) \subset Q^{\prime}$. There exists $c_{1}=c_{1}(\Lambda(Q), \varpi(Q), \varepsilon, \delta)>0$ such that

$$
\mathbb{P}^{x}\left(T_{R}<\tau_{Q}\right) \geq c_{1}, \quad x \in Q^{\prime} .
$$

Proof Note, that

$$
\operatorname{dist}\left(\partial Q, \partial Q^{\prime}\right)=\left|r^{\alpha_{\max } / \alpha_{i}}-k r^{\alpha_{\max } / \alpha_{i}}\right|=\left|\varepsilon \frac{r^{\alpha_{\max } / \alpha_{i}}}{r^{\alpha_{\max } / \alpha_{\min }}}\right| \geq \varepsilon .
$$

Let $x \in Q^{\prime}$ be arbitrary and $\varphi:\left[0, t_{0}\right] \rightarrow \mathbb{R}^{d}$ be a polygonal path such that $\varphi(0)=x$ and $\varphi\left(t_{0}\right)=y$ and the image of $\varphi$ is contained in $Q^{\prime}$. Then the assertion follows by Theorem 3.5 and

$$
\mathbb{P}^{x}\left(\sup _{s \leq t_{o}}\left|X_{s}-\varphi(s)\right|<\varepsilon\right) \leq \mathbb{P}^{x}\left(T_{R}<\tau_{Q}\right) .
$$

Corollary 3.7 Let $r \in(0,1], x_{0} \in \mathbb{R}^{d}$ and $\varepsilon \in\left(0, r^{\alpha_{\max } / \alpha_{\min }} / 4\right)$. For $x \in M_{r}\left(x_{0}\right)$, we define $R:=M_{s}(x)$ such that $R \subset M_{r}\left(x_{0}\right)=: M$ and $\operatorname{dist}(\partial R, \partial M)>\varepsilon$. Then there exists $\xi=\xi(\varepsilon, \Lambda(M), r, \varpi(M) \in(0,1)$ such that

$$
\mathbb{P}^{y}\left(T_{R}<\tau_{M}\right) \geq \xi=\xi(\epsilon) .
$$

for all $y \in M$ with $\operatorname{dist}(y, \partial M)>\varepsilon$.

Proof Follows immediately by Corollary 3.6.

We now prove the main ingredient for the proof of the Hölder regularity. It states, that sets of positive Lebesgue measure are hit with positive probability.

Theorem 3.8 Let $r \in(0,1], x_{0} \in \mathbb{R}^{d}$ and $M:=M_{r}\left(x_{0}\right)$. There exists a nondecreasing function $\varphi:(0,1) \rightarrow(0,1)$ such that

$$
\mathbb{P}^{x}\left(T_{A}<\tau_{M}\right) \geq \varphi(|A|)
$$

for all $x \in M_{r}^{1 / 2}\left(x_{0}\right)$ and all $A \subset M$ with $|A|>0$.

Proof We will follow the proof of Theorem V.7.4 in [2]. Set

$\varphi(\varepsilon)=\inf \left\{\mathbb{P}^{y}\left(T_{A}<\tau_{M_{R}\left(z_{0}\right)}\right): z_{0} \in \mathbb{R}^{d}, R>0, y \in M_{R}^{1 / 2}\left(z_{0}\right),|A| \geq \varepsilon\left|M_{R}\left(z_{0}\right)\right|, A \subset M_{R}\left(z_{0}\right).\right\}$ and

$$
q_{0}:=\inf \{\varepsilon: \varphi(\varepsilon)>0\}
$$


For $\epsilon$ sufficiently large, we know by Corollary $3.6 \varphi(\epsilon)>0$. We suppose $q_{0}>0$, and we will obtain our contradiction.

Since $q_{0}<1$, we can choose $1>q>q_{0}$ such that $\left(q+q^{2}\right) / 2<q_{0}$. Moreover let $\eta:=\left(q-q^{2}\right) / 2, \quad \beta:=\left(\frac{2^{1-d} q r^{-\sum_{i=1}^{d}\left(\alpha_{\max } / \alpha_{i}\right)}}{q+1}\right)^{1 / d} \quad$ and $\rho=\xi\left((1-\beta) r^{\alpha_{\max } / \alpha_{\min }} / 6\right)$,

where $\xi$ is defined as in Corollary 3.7 .

Let $z \in \mathbb{R}^{d}, R \in(0,1], x \in M_{R}^{1 / 2}(z)$ and $A \subset M_{R}(z)$ such that

$$
q-\eta<\frac{|A|}{\left|M_{R}(z)\right|}<q \text { and } \mathbb{P}^{x}\left(T_{A}<\tau_{M_{R}(z)}\right)<\rho \varphi(q)^{2} .
$$

Without loss of generality, set $R=r$ and $z=x_{0}$. Hence

$$
\mathbb{P}^{x}\left(T_{A}<\tau_{M_{r}\left(x_{0}\right)}\right)<\rho \varphi(q)^{2} .
$$

By Proposition 2.2 there exists a set $D \subset M_{r}\left(x_{0}\right)$ such that

$$
|A| \leq q\left|D \cap M_{r}\left(x_{0}\right)\right| .
$$

Since $|A|>(q-\eta)\left|M_{r}\left(x_{0}\right)\right|$,

$$
\left|D \cap M_{r}\left(x_{0}\right)\right| \geq \frac{|A|}{q}>\frac{(q-\eta)\left|M_{r}\left(x_{0}\right)\right|}{q}=\frac{(q+1)\left|M_{r}\left(x_{0}\right)\right|}{2} .
$$

Define $E=D \cap M_{r}^{\beta}\left(x_{0}\right)$. Since

$$
\frac{(q+1)\left|M_{r}^{\beta}\left(x_{0}\right)\right|}{2}=\frac{(q+1) 2^{d} \beta^{d} r^{\sum_{i=1}^{d} \frac{\alpha_{\max }}{\alpha_{i}}}}{2}=q,
$$

we get $|E|>q$. By the definition of $\varphi$, we have $\mathbb{P}^{x}\left(T_{E}<\tau_{M_{r}\left(x_{0}\right)}\right) \geq \varphi(q)$.

We will first show

$$
\mathbb{P}^{y}\left(T_{A}<\tau_{M_{r}\left(x_{0}\right)}\right) \geq \rho \varphi(q) \text { for all } y \in E .
$$

Let $y \in \partial E$, then $y \in \widehat{R}_{i}$ for some $R_{i} \in \mathcal{R}$ and $\operatorname{dist}\left(y, \partial M_{r}\left(x_{0}\right)\right) \geq(1-\beta) r^{\alpha_{\max } \alpha_{\min }}$. Define $R_{i}^{*}$ as the cube with the same center as $R_{i}$ but sidelength half as long. By Corollary 3.7

$$
\mathbb{P}^{y}\left(T_{R_{i}^{*}}<\tau_{M_{r}\left(x_{0}\right)}\right) \geq \rho .
$$

By Proposition 2.2 (3) for all $R_{i} \in \mathcal{R}$

$$
\left|A \cap R_{i}\right| \geq q\left|R_{i}\right|
$$

and therefore

$$
\mathbb{P}^{x_{0}}\left(T_{A \cap R_{i}}<\tau_{M_{r}\left(x_{0}\right)}\right) \geq \varphi(q) \text { for } x_{0} \in R_{i}^{*} .
$$

Using the strong Markov property, we have for all $y \in E$

$$
\begin{aligned}
& \mathbb{P}^{y}\left(T_{A}<\tau_{M_{r}\left(x_{0}\right)}\right) \geq \mathbb{E}^{y}\left[\mathbb{P}^{X_{T_{i}^{*}}}\left(T_{A}<\tau_{R_{i}}\right) ; T_{R_{i}^{*}}<\tau_{M_{r}\left(x_{0}\right)}\right] \\
& \geq \rho \varphi(q) \text {. }
\end{aligned}
$$


Now we get our contradiction by

$$
\begin{aligned}
& \mathbb{P}^{x}\left(T_{A}<\tau_{M_{r}\left(x_{0}\right)}\right) \geq \mathbb{P}^{x}\left(T_{E}<T_{A}<\tau_{M_{r}\left(x_{0}\right)}\right)
\end{aligned}
$$

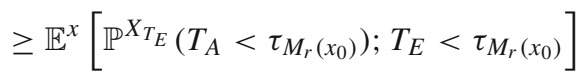

$$
\begin{aligned}
& \geq \rho \varphi(q) \mathbb{P}^{x}\left[T_{E}<\tau_{M_{r}\left(x_{0}\right)}\right] \geq \rho \varphi(q)^{2} .
\end{aligned}
$$

\section{Proof of Theorem 1.2}

In this section we prove our main result.

Proof Let $S:=M_{S}(y) \subset M_{r}\left(x_{0}\right)$ and $A \subset S$ such that $3|A| \geq|S|$. Those sets will be specified later. Set $k=1-\left(\varepsilon / r^{\alpha_{\max } / \alpha_{\min }}\right)$ and $S^{\prime}:=M_{S}^{k}(y)$, where $\varepsilon$ is chosen such that $6\left|S \backslash S^{\prime}\right|=|S|$. Then

$$
6\left|A \cap S^{\prime}\right| \geq|S|
$$

Let $\mathcal{R}$ be a collection of $N$ equal sized rectangles as in Definition 1.1 with disjoint interiors and $\mathcal{R} \subset S$. Moreover let $\mathcal{R}$ to be a covering of $S^{\prime}$. For at least one rectangle $Q \in \mathcal{R}$

$$
6\left|A \cap S^{\prime} \cap Q\right| \geq|Q| \text {. }
$$

Let $Q^{\prime}$ be the rectangle with the same center as $Q$ but each sidelength half as long. By Corollary 3.6 there exists a $c_{2}>0$ such that

$$
\mathbb{P}^{x}\left(T_{Q^{\prime}}<\tau_{S}\right) \geq c_{2}, \quad x \in M_{S}^{1 / 2}(y) .
$$

Using Theorem 3.8 and the strong Markov property there exists a constant $c_{3}>0$ with

$$
\mathbb{P}^{x}\left(T_{A}<\tau_{S}\right) \geq c_{3}, \quad x \in M_{S}^{1 / 2}(y) .
$$

Let $R \geq 2 r$. By Proposition 2.5 there exists a $c_{4}>0$ such that

$$
\mathbb{P}^{z}\left(X_{\tau_{M_{r}\left(x_{0}\right)}} \notin M_{R}\left(x_{0}\right)\right) \leq c_{4}\left(\frac{r}{R}\right)_{\max }^{\alpha} \text { for all } z \in M_{r}\left(x_{0}\right) .
$$

Let

$$
\gamma:=\left(1-c_{3}\right), \quad \rho:=\left(\frac{c_{3} \gamma^{2}}{4 c_{4}}\right)^{1 / \alpha_{\max }} \wedge\left(\frac{\gamma}{2}\right)^{1 / \alpha_{\max }} \text { and } \beta:=\frac{\log (\gamma)}{\log (\rho)} .
$$

By linearity it suffices to suppose $0 \leq h \leq M$ on $\mathbb{R}^{d}$. We first consider the case $r=1$.

Let $M_{i}=M_{\rho^{i}}\left(x_{0}\right)$ and $\tau_{i}=\tau_{M_{i}}$. We will show that for all $k \in \mathbb{N}_{0}$

$$
\underset{M_{k}}{\operatorname{osc}} h:=\sup _{M_{k}} h-\inf _{M_{k}} h \leq M \gamma^{k} .
$$

To shorten notation, we set $a_{i}=\inf _{M_{i}} h$ and $b_{i}=\sup _{M_{i}} h$. Assertion (4.3) will be will be proved by induction. Let $k \in \mathbb{N}$ be arbitrary but fixed. We suppose $b_{i}-a_{i} \leq M \gamma^{i}$ for all $i \leq k$; then we need to show

$$
b_{k+1}-a_{k+1} \leq M \gamma^{k+1}
$$


By definition $M_{k+1} \subset M_{k}$ and therefore in particular $a_{k} \leq h \leq b_{k}$ on $M_{k+1}$. Set

$$
A^{\prime}=\left\{z \in M_{k+1}: h(z) \leq\left(a_{k}+b_{k}\right) / 2\right\} .
$$

Without loss of generality, assume $2\left|A^{\prime}\right| \geq\left|M_{k+1}\right|$. If this assumption does not hold, we consider $M-h$ instead of $h$. Let $A \subset A^{\prime}$ be compact such that $3|A| \geq\left|M_{k+1}\right|$. By (4.2) there exists a $c_{3}>0$ such that $\mathbb{P}^{y}\left(T_{A}<\tau_{k}\right) \geq c_{3}$ for all $y \in M_{k+1}$.

Let $\varepsilon>0$ and $y, z \in M_{k+1}$ such that $h(y) \geq b_{k+1}-\varepsilon$ and $h(z) \leq a_{k+1}+\epsilon$. Since $h$ is harmonic, $h\left(X_{t}\right)$ is a martingale. We get by optimal stopping

$$
\begin{aligned}
h(y)-h(z)= & \mathbb{E}^{y}\left(h\left(X_{T_{A}}\right)-h(z) ; \tau_{k}>T_{A}\right) \\
& +\mathbb{E}^{y}\left(h\left(X_{\tau_{k}}\right)-h(z) ; \tau_{k}<T_{A}, X_{\tau_{k}} \in M_{k-1}\right) \\
& +\sum_{i=1}^{\infty} \mathbb{E}^{y}\left(h\left(X_{\tau_{k}}\right)-h(z) ; \tau_{k}<T_{A}, X_{\tau_{k}} \in M_{k-i-1} \backslash M_{k-i}\right) .
\end{aligned}
$$

We will now study these three components on the right hand side seperately. Note $h(z) \geq$ $a_{k} \geq a_{k-i-1}$ for all $i \in \mathbb{N}$

(1) In the first component, $X$ enters $A \subset A^{\prime}$ before leaving $M_{k}$. Hence

$$
\begin{array}{r}
\mathbb{E}^{y}\left(h\left(X_{T_{A}}\right)-h(z) ; T_{A}<\tau_{k}\right) \leq \mathbb{E}^{y}\left(\frac{a_{k}+b_{k}}{2}-a_{k} ; T_{A}<\tau_{k}\right) \\
=\frac{b_{k}-a_{k}}{2} \mathbb{P}^{y}\left(T_{A}<\tau_{k}\right) \leq \frac{M \gamma^{k}}{2} \mathbb{P}^{y}\left(T_{A}<\tau_{k}\right) .
\end{array}
$$

(2) In the component $X$ leaves $M_{k}$ before entering $A$. While leaving $M_{k}, X$ does not make a big jump in the following sense: $X$ is at time $\tau_{k}$ in $M_{k-1}$. Hence in this case $h\left(X_{\tau_{k}}\right) \leq$ $b_{k-1}$. This yields to

$$
\begin{aligned}
\mathbb{E}^{y} & \left(h\left(X_{\tau_{k}}\right)-h(z) ; \tau_{k}<T_{A}, X_{\tau_{k}} \in M_{k-1}\right) \leq \mathbb{E}^{y}\left(b_{k-1}-a_{k-1} ; \tau_{k}<T_{A}, X_{\tau_{k}} \in M_{k-1}\right) \\
& =\left(b_{k-1}-a_{k-1}\right) \mathbb{P}^{y}\left(\tau_{k}<T_{A}\right) \leq M \gamma^{k-1}\left(1-\mathbb{P}^{y}\left(T_{A}<\tau_{k}\right)\right) .
\end{aligned}
$$

(3) In the third component $X_{\tau_{k}} \in M_{k-i-1}$ for $i \in \mathbb{N}$. Therefore $h\left(\tau_{k}\right) \leq b_{k-i-1}$.

$$
\begin{aligned}
& \sum_{i=1}^{\infty} \mathbb{E}^{y}\left(h\left(X_{\tau_{k}}\right)-h(z) ; \tau_{k}<T_{A}, X_{\tau_{k}} \in M_{k-i-1} \backslash M_{k-i}\right) \\
& \quad \leq \sum_{i=1}^{\infty} \mathbb{E}^{y}\left(\left(b_{k-i-1}-a_{k-i-1}\right) ; \tau_{k}<T_{A}, X_{\tau_{k}} \in M_{k-i-1} \backslash M_{k-i}\right) \\
& \quad=\sum_{i=1}^{\infty}\left(b_{k-i-1}-a_{k-i-1}\right) \mathbb{P}^{y}\left(X_{\tau_{k}} \notin M_{k-i}\right) \leq \sum_{i=1}^{\infty} M \gamma^{k-i-1} c_{4}\left(\frac{\rho^{k}}{\rho^{k-i}}\right)^{\alpha_{\max }} \\
& \quad=c_{4} M \gamma^{k-1} \sum_{i=1}^{\infty}\left(\frac{\rho^{\alpha_{\max }}}{\gamma}\right)^{i}=c_{4} M \gamma^{k-1} \frac{\rho^{\alpha_{\max }}}{\gamma-\rho^{\alpha_{\max }}} \\
& \quad \leq 2 c_{4} \gamma^{k-2} M \rho^{\alpha_{\max }} \leq \frac{c_{3} M \gamma^{k}}{2},
\end{aligned}
$$

where we used

$$
\frac{\rho^{\alpha_{\max }}}{\gamma} \leq \frac{1}{2} \text { and } \rho \leq\left(\frac{\gamma}{2}\right)^{1 / \alpha_{\max }} \Leftrightarrow \frac{\rho^{\alpha_{\max }}}{\gamma-\rho^{\alpha_{\max }}} \leq 2 \frac{\rho^{\alpha_{\max }}}{\gamma} .
$$


Note, that the choice of $\gamma=1-c_{3}$ implies $\frac{\gamma-2}{2 \gamma} c_{3}+\frac{1}{\gamma}+\frac{c_{3}}{2}=\gamma$. Hence

$$
\begin{aligned}
h(y)-h(z) & \leq \frac{M \gamma^{k}}{2} \mathbb{P}^{y}\left(T_{A}<\tau_{k}\right)+\frac{M \gamma^{k-1}}{r^{\beta}}\left(1-\mathbb{P}^{y}\left(T_{A}<\tau_{k}\right)\right)+\frac{c_{3} M \gamma^{k}}{2} \\
& =M \gamma^{k}\left(\left(\frac{\gamma-2}{2 \gamma} \mathbb{P}^{y}\left(T_{A}<\tau_{k}\right)+\frac{1}{\gamma}+\frac{c_{3}}{2}\right)\right. \\
& \leq M \gamma^{k}\left(\frac{\gamma-2}{2 \gamma} c_{3}+\frac{1}{\gamma}+\frac{c_{3}}{2}\right) \\
& =M \gamma^{k+1} .
\end{aligned}
$$

We conclude that

$$
b_{k+1}-a_{k+1} \leq M \gamma^{k+1}+2 \varepsilon .
$$

Since $\varepsilon$ is arbitrary, this proves (4.4) and therefore (4.3). Let $x, y \in M_{1}\left(x_{0}\right)$ and choose $k \in \mathbb{N}_{0}$ such that $M_{\rho^{k}}^{2}(x)$ is the smallest rectangle with $y \in M_{\rho^{k}}(x)$.

Then $|x-y| \leq 2 \sqrt{d} \rho^{k}$ and therefore

$$
k \geq \frac{\log \left(\frac{|x-y|}{2 \sqrt{d}}\right)}{\log (\rho)} \quad \text { for } y \in M_{\rho^{k}}(x) .
$$

Hence

$$
\begin{aligned}
|h(y)-h(x)| & \leq M \gamma^{k}=M e^{k \log (\gamma)} \\
& \leq M e^{\left(\log [|x-y| /(2 \sqrt{d}))\left(\left(\alpha_{\max } \log (\gamma)\right) /(\alpha \log (\rho))\right)\right.} \\
& =\frac{M|x-y|^{\log (\gamma) / \log (\rho)}}{2 \sqrt{d}}=c_{5} M|x-y|^{\beta} .
\end{aligned}
$$

Now let $h$ be harmonic on $M_{r}^{2}\left(x_{0}\right)$. Then $h^{\prime}(x):=h\left(r^{\alpha_{\max } / \alpha_{\min }} x\right)$ is harmonic on $M_{1}^{2}\left(x_{0}\right)$. Let $x, y \in M_{1}\left(x_{0}\right)$ and $x^{\prime}, y^{\prime} \in M_{r}\left(x_{0}\right)$ such that

$$
x=\left(x_{1}^{\prime} / r^{\alpha_{\max } / \alpha_{1}}, \ldots, x_{d}^{\prime} / r^{\alpha_{\max } / \alpha_{d}}\right), \quad y=\left(y_{1}^{\prime} / r^{\alpha_{\max } / \alpha_{1}}, \ldots, y_{d}^{\prime} / r^{\alpha_{\max } / \alpha_{d}}\right) \in M_{1}\left(x_{0}\right) .
$$

Then $|x-y| \leq r^{\alpha_{\max } / \alpha_{\min }}\left|x^{\prime}-y^{\prime}\right|$. Set $\tilde{x}=x^{\prime} / r^{\alpha_{\max } / \alpha_{\min }}$ and $\tilde{y}=y^{\prime} / r^{\alpha_{\max } / \alpha_{\min }}$. We conclude

$$
\begin{aligned}
\left|h\left(x^{\prime}\right)-h\left(y^{\prime}\right)\right| & =\left|h\left(r^{\alpha_{\max } / \alpha_{\min }} \tilde{x}\right)-h\left(r^{\alpha_{\max } / \alpha_{\min }} \tilde{y}\right)\right|=\left|h^{\prime}(\tilde{x})-h^{\prime}(\tilde{y})\right| \leq c_{1}|\tilde{x}-\tilde{y}|^{\beta} \sup _{\tilde{z} \in \mathbb{R}^{d}}|h(\tilde{z})| \\
& =c_{1}\left|\frac{x^{\prime}}{r^{\alpha_{\max } / \alpha_{\min }}}-\frac{y^{\prime}}{r^{\alpha_{\max } / \alpha_{\min }}}\right|^{\beta} \sup _{z \in \mathbb{R}^{d}}|h(z)|=c_{1}\left(\frac{\left|x^{\prime}-y^{\prime}\right|}{r^{\alpha_{\max } / \alpha_{\min }}}\right)^{\beta} \sup _{z \in \mathbb{R}^{d}}|h(z)| .
\end{aligned}
$$

\section{Acknowledgements Open Access funding provided by Projekt DEAL.}

Open Access This article is licensed under a Creative Commons Attribution 4.0 International License, which permits use, sharing, adaptation, distribution and reproduction in any medium or format, as long as you give appropriate credit to the original author(s) and the source, provide a link to the Creative Commons licence, and indicate if changes were made. The images or other third party material in this article are included in the article's Creative Commons licence, unless indicated otherwise in a credit line to the material. If material is not included in the article's Creative Commons licence and your intended use is not permitted by statutory regulation or exceeds the permitted use, you will need to obtain permission directly from the copyright holder. To view a copy of this licence, visit http://creativecommons.org/licenses/by/4.0/. 


\section{References}

1. Barles, G., Chasseigne, E., Imbert, C.: Hölder continuity of solutions of second-order non-linear elliptic integro-differential equations. J. Eur. Math. Soc. (JEMS) 13(1), 1-26 (2011)

2. Bass, R.F.: Diffusions and elliptic operators. Probability and its Applications (New York). Springer-Verlag, New York (1998)

3. Bass, R.F., Chen, Z.Q.: Systems of equations driven by stable processes. Probab. Theory Relat. Fields 134(2), 175-214 (2006)

4. Bass, R.F., Chen, Z.-Q.: Regularity of harmonic functions for a class of singular stable-like processes. Math. Z. 266(3), 489-503 (2010)

5. Bass, R.F., Kassmann, M.: Hölder continuity of harmonic functions with respect to operators of variable order. Comm. Partial Differ. Equ. 30(7-9), 1249-1259 (2005)

6. Bass, R.F., Levin, D.A.: Harnack inequalities for jump processes. Potential Anal. 17(4), 375-388 (2002)

7. Bogdan, K., Sztonyk, P., Knopova, V.: Heat kernel of anisotropic nonlocal operators (2017). arXiv: 1704.03705

8. Caffarelli, L., Silvestre, L.: An extension problem related to the fractional Laplacian. Comm. Partial Differ. Equ. 32(8), 1245-1260 (2007)

9. Caffarelli, L., Silvestre, L.: Regularity theory for fully nonlinear integro-differential equations. Comm. Pure Appl. Math. 62(5), 597-638 (2009)

10. Caffarelli, L.A., Leitão, R., Urbano, J.M.: Regularity for anisotropic fully nonlinear integro-differential equations. Math. Ann. 360(3-4), 681-714 (2014)

11. Chaker, J.: The martingale problem for a class of nonlocal operators of diagonal type. Math. Nachr. 292(11), 2316-2337 (2019)

12. Chaker, J., Kassmann, M.: Nonlocal operators with singular anisotropic kernels. Comm. Partial Differ. Equ. 45(1), 1-31 (2020)

13. Chaker, J., Kassmann, M., Weidner, M.: Robust Hölder Estimates for Parabolic Nonlocal Operators (2019). arXiv:1912.09919

14. Cont, R., Tankov, P.: Financial modelling with jump processes. Chapman \& Hall/CRC Financial Mathematics Series. Chapman \& Hall/CRC, Boca Raton (2004)

15. De Giorgi, E.: Sulla differenziabilità e l'analiticità delle estremali degli integrali multipli regolari. Mem. Accad. Sci. Torino. Cl. Sci. Fis. Mat. Nat. 3(3), 25-43 (1957)

16. Ethier, S.N., Kurtz, T.G.: Markov processes. Wiley Series in Probability and Mathematical Statistics: Probability and Mathematical Statistics. Characterization and convergence. John Wiley \& Sons, Inc., New York (1986)

17. Fangjun, X.: A class of singular symmetric Markov processes. Potential Anal. 38(1), 207-232 (2013)

18. Friesen, M., Jin, P., Rüdiger, B.: Existence of densities for stochastic differential equations driven by Lévy processes with anisotropic jumps (2018). arXiv: 1810.07504

19. Gilbarg, D., Trudinger, N.S.: Elliptic partial differential equations of second order. Classics in Mathematics. Springer-Verlag, Berlin (2001) Reprint of the edition (1998)

20. Kassmann, M.: Harnack inequalities: an introduction. Bound. Value Probl., Art. ID 81415, 21 (2007)

21. Kassmann, M., Kim, K.-Y., Kumagai, T.: Heat kernel bounds for nonlocal operators with singular kernels (2019). arXiv:1910.04242

22. Kassmann, M., Rang, M., Schwab, R.W.: Integro-differential equations with nonlinear directional dependence. Indiana Univ. Math. J. 63(5), 1467-1498 (2014)

23. Krylov, N.V., Safonov, M.V.: An estimate for the probability of a diffusion process hitting a set of positive measure. Dokl. Akad. Nauk SSSR 245(1), 18-20 (1979)

24. Kulczycki, T., Ryznar, M.: Transition density estimates for diagonal systems of SDEs driven by cylindrical $\alpha$-stable processes. ALEA Lat. Am. J. Probab. Math. Stat. 15(2), 1335-1375 (2018)

25. Kulczycki, T., Ryznar, M.: Semigroup properties of solutions of SDEs driven by lévy processes with independent coordinates (2019). arXiv:1906.07173

26. Kulczycki, T., Ryznar, M., Sztonyk, P.: Strong feller property for SDEs driven by multiplicative cylindrical stable noise (2018). arXiv: 1811.05960

27. Lara, H.C., Gonzalo, D.: Regularity for solutions of non local parabolic equations. Calc. Var. Partial Differ. Equ. 49(1-2), 139-172 (2014)

28. Moser, J.: On Harnack's theorem for elliptic differential equations. Comm. Pure Appl. Math. 14, 577-591 (1961)

29. Nash, J.: Parabolic equations. Proc. Nat. Acad. Sci. USA 43, 754-758 (1957)

30. Sato, K.: Lévy processes and infinitely divisible distributions. Cambridge Studies in Advanced Mathematics, vol. 68. Cambridge University Press, Cambridge (1999). Translated from the 1990 Japanese original, Revised by the author 
31. Silvestre, L.: Hölder estimates for solutions of integro-differential equations like the fractional Laplace. Indiana Univ. Math. J. 55(3), 1155-1174 (2006)

32. Song, R., Vondraček, Z.: Harnack inequality for some classes of Markov processes. Math. Z. 246(1-2), 177-202 (2004)

33. Wang, L., Zhang, X.: Harnack inequalities for SDEs driven by cylindrical $\alpha$-stable processes. Potential Anal. 42(3), 657-669 (2015)

Publisher's Note Springer Nature remains neutral with regard to jurisdictional claims in published maps and institutional affiliations. 